\title{
Nature-inspired passive and active kinematics of a flapping foil
}

Junyoung Kim, Junshin Park and Donghyun You

Flow Physics and Engineering Laboratory - Pohang University of Science and Technology (POSTECH)

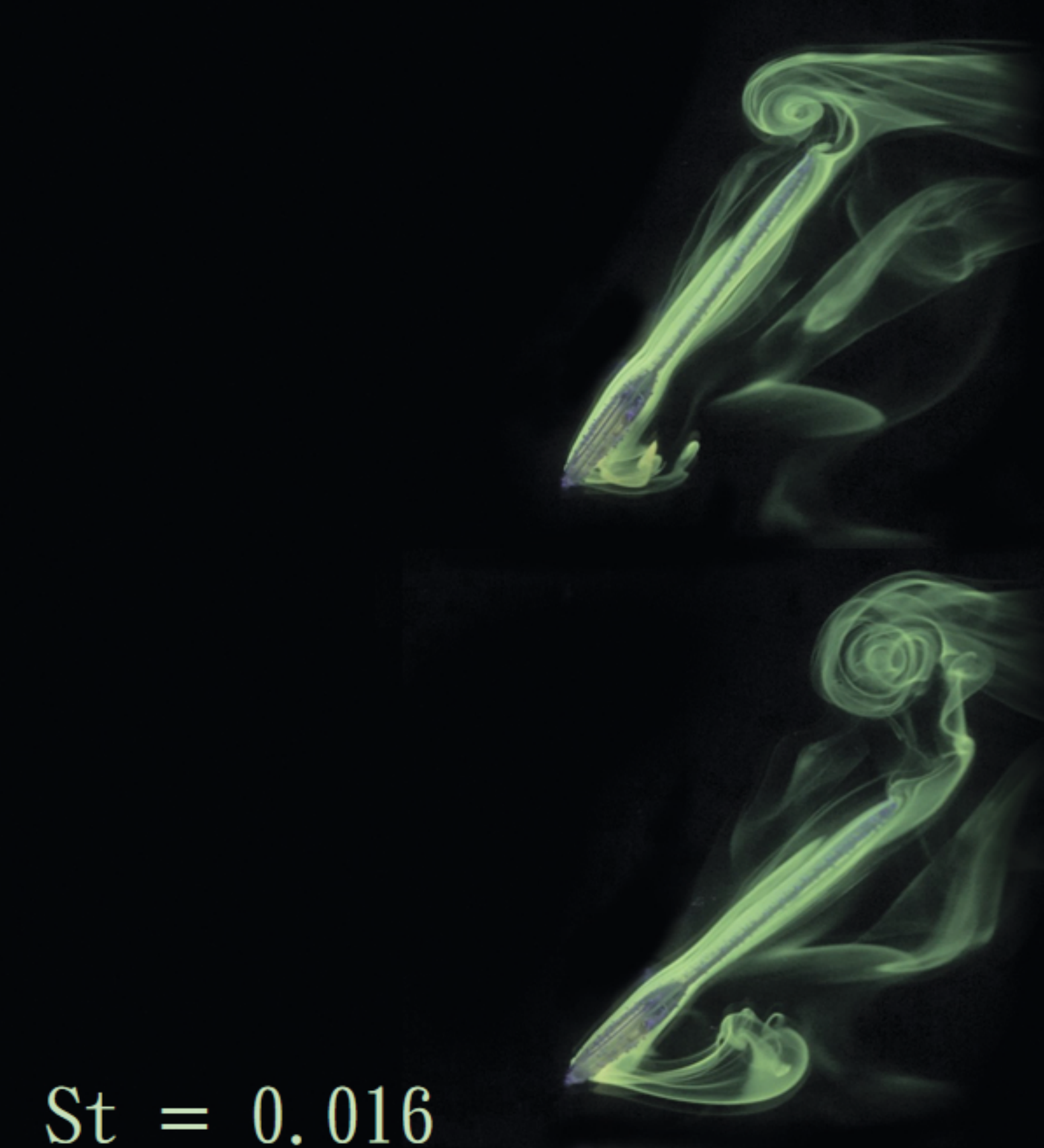

$$
\text { St }=0.080
$$
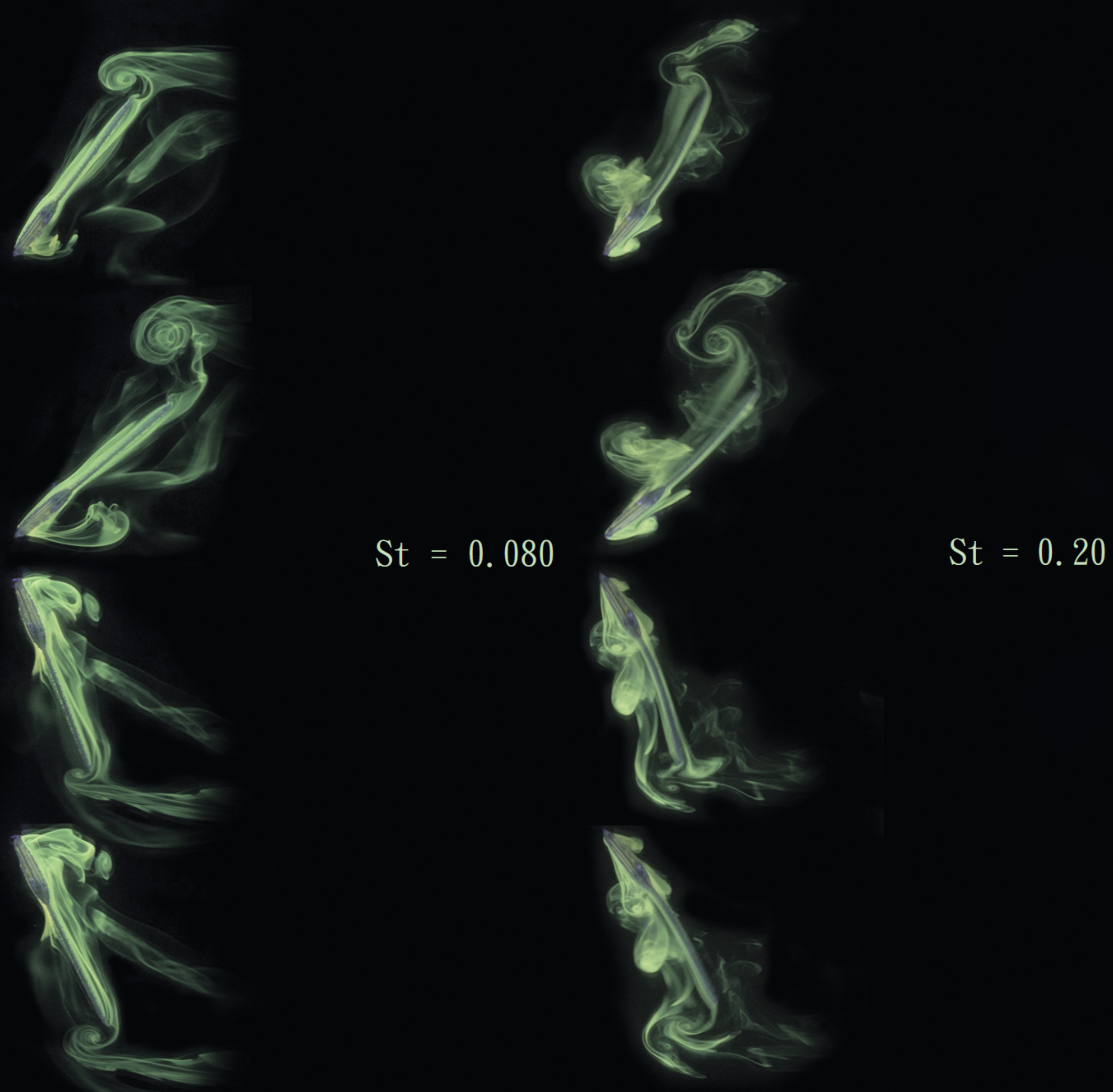

St $=0.20$
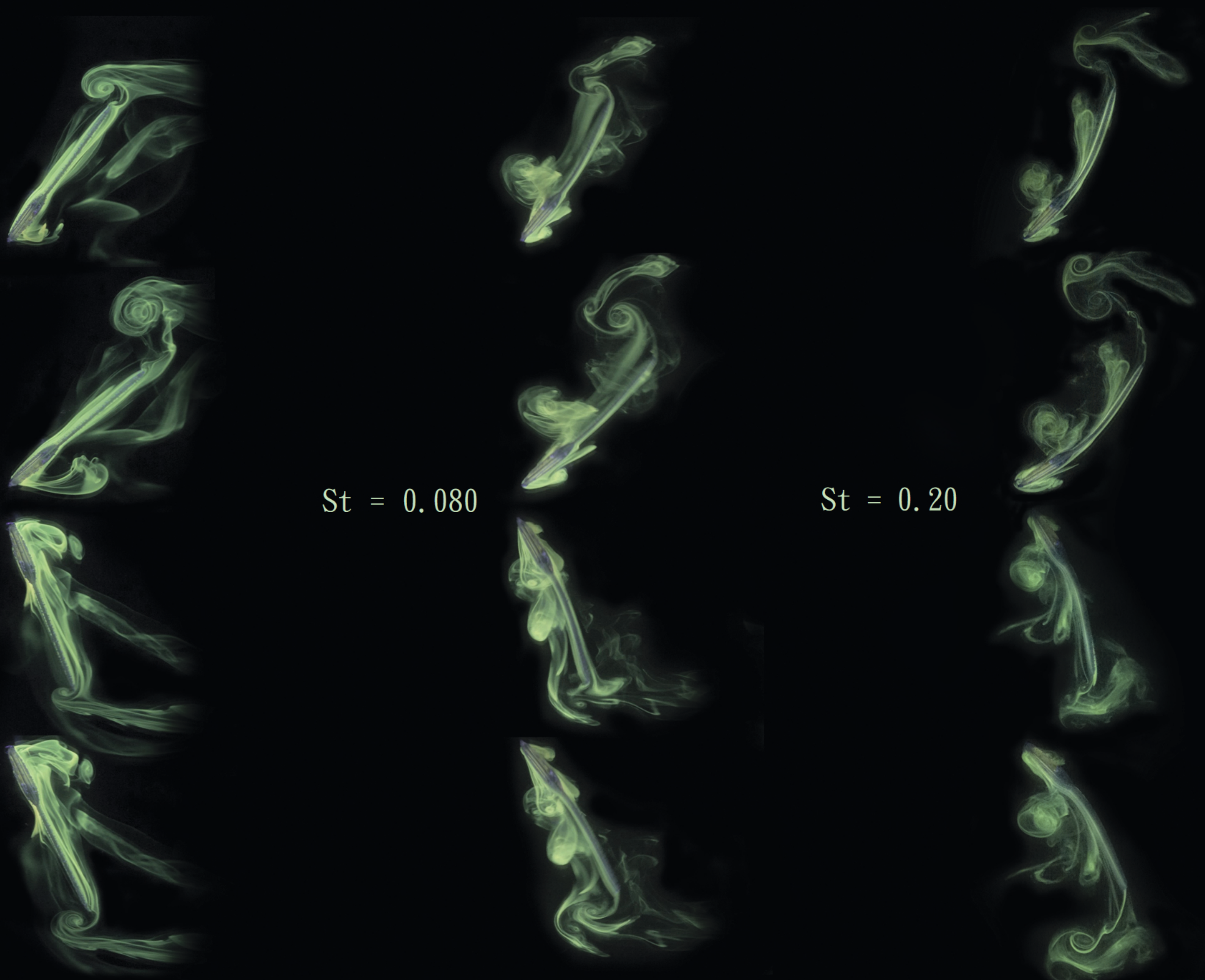

Carangiform tails take a major role in generation of the total thrust of aquatic animals. Carangiform locomotion is modeled as heaving and pitching motions of a flexible foil, and dye visualizations are performed at Reynolds number of $3850\left(\operatorname{Re}=\mathrm{U}_{\infty} \mathrm{C} / \mathrm{v}\right)$. Flexibility effects on wake structure are illustrated with various Strouhal numbers $\left(\mathrm{St}=\mathrm{fc} / \mathrm{U}_{\infty}\right)$. 